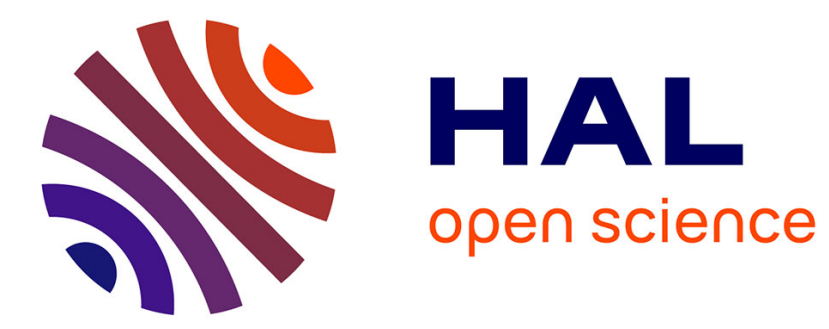

\title{
Twisters: an analogy of bilayers for twisting
}

Sébastien Turcaud, Anders Thorin, Yves Bréchet, Peter Fratzl, John J. Dunlop

\section{To cite this version:}

Sébastien Turcaud, Anders Thorin, Yves Bréchet, Peter Fratzl, John J. Dunlop. Twisters: an analogy of bilayers for twisting. 2019. hal-02076495

\section{HAL Id: hal-02076495 \\ https://hal.science/hal-02076495}

Preprint submitted on 22 Mar 2019

HAL is a multi-disciplinary open access archive for the deposit and dissemination of scientific research documents, whether they are published or not. The documents may come from teaching and research institutions in France or abroad, or from public or private research centers.
L'archive ouverte pluridisciplinaire HAL, est destinée au dépôt et à la diffusion de documents scientifiques de niveau recherche, publiés ou non, émanant des établissements d'enseignement et de recherche français ou étrangers, des laboratoires publics ou privés. 


\title{
Twisters: an analogy of bilayers for twisting
}

\author{
Sébastien TuRCAUD ${ }^{1,2}$, Anders ThORIN ${ }^{3}$, Yves BrÉCHET ${ }^{2}$, Peter FRATZL ${ }^{1}$, John DunLOP ${ }^{1}$ \\ ${ }^{1}$ Max Planck Institute of Colloids and Interfaces, Biomaterials Department, Potsdam, Germany \\ 2 Materials and Processes Science and Engineering Laboratory, Saint-Martin-d'Hères, France \\ 3 Department of Mechanical Engineering, McGill University, Montreal, Canada
}

ABSTRACT Benders, such as bilayers, are well-known shape-changing structures which bend upon activation by a stimulus, such as temperature. The objective of this contribution is to propose new shape-changing rod-like structures, referred to as twisters, which twist upon activation. A simple biomimetic design principle based on symmetry considerations is proposed and used to derive a mechanical model. The kinematics of the response of the twister to the simulus is inspired from FEM simulations, and slightly simplified to allow for an analytical resolution of the governing equations. Proposed twisters are shown to first stretch as the stimulus increases until a bifurcation is reached, then, they undergo a mixed stretching-twisting regime. An accurate approximation of the bifurcation point is derived and serves as a guideline for the design of twisters in accordance with chosen specifications. Results are illustrated using twisters with three different behaviours. The methodology can be straightfowardly extended to more complex kinematics and other constitutive laws.

Introduction Nature has a long experience in designing mechanical systems, offering a great source of inspiration for human-made constructions. Of particular interest in this work is the phenomena of shape change in response to an externally changing stimulus, typically humidity, present in a variety of natural systems [6]. The underlying design principle consists in the structure-function relationship of a system possessing heterogeneous expansion properties distributed throughout its shape, leading to differential expansion upon a variety of external stimuli (activation). This differential expansion is responsible for an overall shape change, which is referred to as morphing.

Among the numerous morphers present in Nature, bilayers are probably the simplest. For example, pinecones open and close depending on air moisture to release their seeds at a time favorable for their germination [5]. Metallic bilayers [11] are a well-known engineering system based on this design principle: they display bending upon activation by change of temperature (benders). Intrigued by twisting motions of some natural morphers such as the twist-like opening of seed pods [2] and the coiling actuation of the stork's bill awn [1], here we investigate an anologous design principle to benders leading to twisting motions activated by an external stimulus. Systems exhibiting such behaviours are referred to as twisters; they are to twisting what benders are to bending.

In a previous paper [12], the design of biphasic extrudable morphers following Curie's principle regarding symmetry conservation was explored using the finite-element method and revealed twisting structures. Here, a particular rotationally symmetric material architecture is more thorougly investigated. The proposed twisters derive from qualitative observations of twisting structures simulated using the finite element method (section 1). These observations lead to a simple kinematical description which is used to propose an analytical model of twisters based on differential theory and elastic rod theory (section 2). Using the analytical methods derived in section 3, the mechanical model is shown to capture the stretching-twisting behavior of these twisters (section 4). Several illustrations are provided together with a sensitivity analysis based on approximate equations.

Description of twisters and qualitative analysis with FEM We focus on translationally invariant rod-like structures (cylinders) made of two different linear elastic materials subjected to differential eigenstrains. Eigenstrains do not generate any stresses per se, but they may 
lead to elastic strains because of geometric compatibility, indirectly causing elastic stresses [7]. The eigenstrains are assumed to have a linear dependency w.r.t. an externally applied stimulus, which is chosen to be constant throughout the structure.

The cross-sections are made out of a regular $n$-sided polygon (the core) with wings of the same shape but smaller size, placed on each side as depicted in fig. 1. The core has a low expansion coefficient $\left(\alpha_{\mathrm{c}}\right)$ while the wings have a high expansion coefficient $\left(\alpha_{\mathrm{w}}\right)$, thus leading to differential expansion. The circumradii of the $n$-sided polygons are denoted by $R_{\mathrm{c}}$ for the core and $R_{\mathrm{w}}$ for each wing. Such structures are referred to as $n$-twisters.

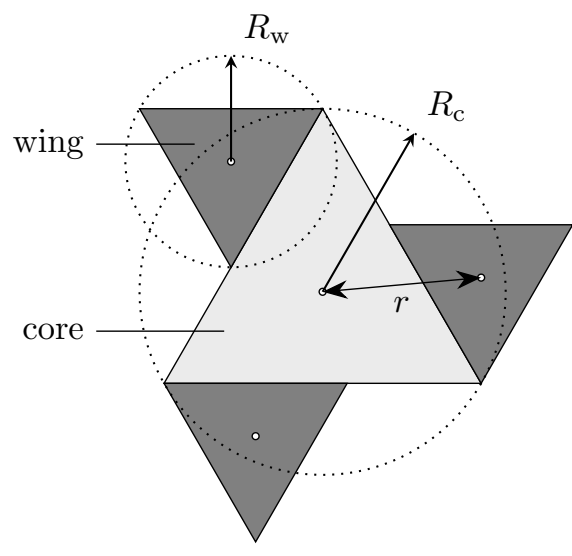

(a) 3-twister

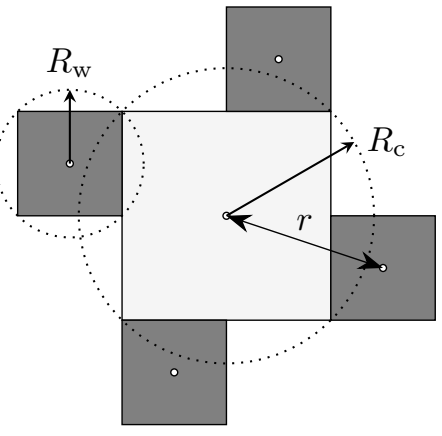

(b) 4-twister

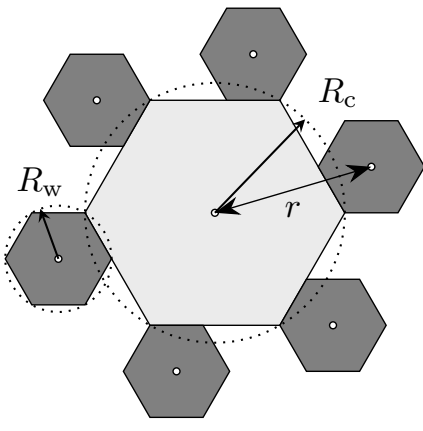

(c) 6-twister

Figure 1: Rotationally symmetric concave cross-sections of twisters made out of regular $n$-sided polygons ( $n=3,4,6)$. (light gray) core with a low expansion coefficient, (dark gray) wings with a high expansion coefficient.

The rationale behind such shapes is Curie's symmetry conservation principle: "effects have at least the symmetry of their causes" [4]. In 2D, all orthogonal symmetries can be generated from mirror and rotational symmetries. Bending conserves mirror symmetry (bending plane) and benders typically possess a mirror plane. Similarly, twisting conserves rotational symmetry (rotational axis), hence the idea to look at potentially twisting structures already possessing rotational symmetry. Additionally, being translationally invariant, such structures possess the benefit of being extrudable and so are easy to process on an industrial scale.

The finite elements method was used in Abaqus ${ }^{\circledR}$ to observe the behaviour of twisters under the action of a thermal stimulus (see fig. 2 left), under free-free boundary conditions [12]. Considering large transformations (option NLgeom), the linearly elastic structure was statically loaded by a thermal scalar field, generating differential expansion. The adaptive mesh was composed of about ten thousand linear 8-node brick elements (C3D8). As the stimulus $S$ increased, the differential expansion between the wings and the core grew. Interestingly, the morphing featured a twisting instability. For small values of $S$, the twister only stretched; but when $S$ became larger than a critical value $S_{\text {crit }}$, its morphing became a combination of stretching and twisting $\left(S>S_{\text {crit }}\right.$ ).

The following section is dedicated to the derivation of an analytical model for the above-described twisters, whose kinematics is inspired by the qualitative observation of the FEM simulations.

The chosen kinematic to describe the twisters deformation are derived in the framework of large strains and large displacements. Even though the methodology can be straightforwardly applied to other constitutive laws, it is however assumed that the materials of both core and wings are linear 
FEM observations

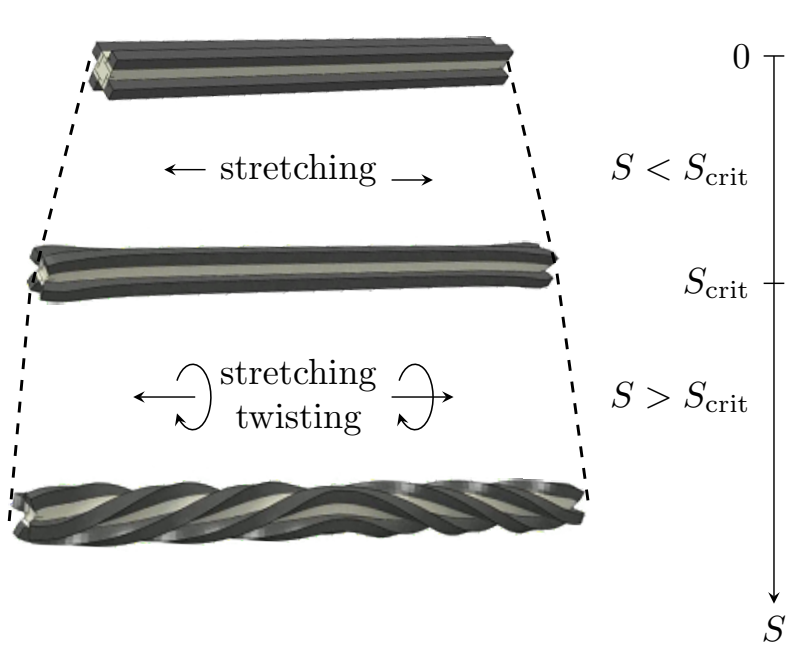

Kinematical scenario

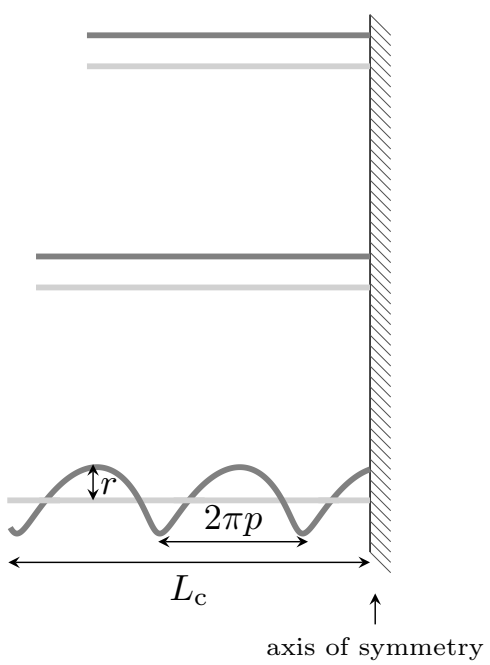

Figure 2: Kinematical scenario based on FEM observations of the stretching-twisting instability of twisters as a function of $S$. (Left) Morphing stages of a 4-twister using FEM. (Right) Center lines of the core (light) and one wing (dark) in the two stages of the kinematical scenario. In the second stage ( $S>S_{\text {crit }}$ ), the wings helically wind around the core.

elastic and undergo small strains, hence their constitutive law is given by a simple Hooke's law. The chosen kinematical scenario (see fig. 2 right) is such that:

- the core is allowed to undergo stretching and torsion, but no bending;

- the wings are constrained to wind around the core in a helical manner ${ }^{1}$ and can thus undergo stretching as well as a combination of bending and torsion.

The center line of the wings thus follows a helix of constant radius $r$ and constant pitch $2 \pi p$ which winds around the twisted (but straight) center line of the core. The assumption that the distance between the centroids of the core and wings $r$ is constant derives from the fact that we only impose longitudinal expansions and neglect dimensional changes in the plane of the cross-section (e.g. due to Poisson effect), as well as warping effects. More questionable is the assumption of constant pitch: in the bottom simulation of fig. 2, the twister exhibits not only i) a varying twist, but also ii) a change of sign of the twist. Noting that ii) is due to the mirror symmetry of the simulation with free-free boundary conditions, we here consider only a half-twister or, equivalently, a free-fixed twister. Regarding i), simplicity is favoured over accuracy: all the following methodology can be adapted to include a varying twist, but it would add unnecessary complications to the equations.

The elastic energy of one rod of length $L$ with imposed longitudinal eigenstrain $\left(\epsilon^{*}\right)$ undergoing a combination of stretching $(\epsilon)$, single bending $(\kappa)$ and torsion $(\tau)$ is given by:

$$
\mathcal{E}_{S}(\epsilon, \kappa, \tau)=\frac{L}{2}(\underbrace{E A\left(\epsilon-\epsilon^{*}\right)^{2}}_{\text {stretching }}+\underset{\text { bending }}{E I \kappa^{2}}+\underbrace{\mu J \tau^{2}}_{\text {torsion }})
$$

where $E$ and $\mu$ are the tensile and shear moduli of its material and $A, I, J$ the area, second moment

${ }^{1}$ Chirality of twisters is absent from our analytical description, hence negative and positive $\bar{\tau}$ are equivalent (handiness of helices). In practice, the twister twists in the direction determined by its initial chirality and thus "selects" one branch in the $(\bar{\epsilon}, \bar{\tau})$ space. 


\begin{tabular}{|c|c|c|c|}
\hline \multicolumn{2}{|c|}{ Nomenclature } & $\kappa_{\mathrm{c}}, \kappa_{\mathrm{w}}$ & Curvature of core, wing \\
\hline \multicolumn{2}{|c|}{ Dimensionless parameters } & $L_{\mathrm{c}}, L_{\mathrm{w}}$ & Length of core, wing \\
\hline$A_{0}$ & & & Number of wings \\
\hline$A_{0}$ & $\begin{array}{l}\text { Area coemcient } \\
\text { Inertia coefficient }\end{array}$ & $R_{\mathrm{c}}, R_{\mathrm{w}}$ & Circumradius of core, wing \\
\hline$I_{0}$ & Inertia co & $\tau_{\mathrm{c}}, \tau_{\mathrm{w}}$ & Torsion of core, wing \\
\hline$\frac{J_{0}}{\bar{F}}$ & $\begin{array}{ll}101 \\
\text { Stit }\end{array}$ & $L_{0}$ & Length of twister at rest \\
\hline $\bar{\epsilon}, \bar{\tau}$ & Dimensionless strain and torsion of core & $r$ & $\begin{array}{l}\text { Distance between centroids of core and } \\
\text { wings }\end{array}$ \\
\hline$\overline{\mathcal{E}}_{S}$ & Dimensionless energy & \multicolumn{2}{|c|}{ Material parameters } \\
\hline$\overline{\kappa_{\mathrm{w}}}, \overline{\tau_{\mathrm{w}}}$ & Dimensionless curvature, torsion of wing & $\alpha_{\mathrm{c}}, \alpha_{\mathrm{w}}$ & Expansion coefficient of core, wing \\
\hline $\bar{R}$ & Size ratio & $\Delta \alpha$ & The differential expansion coefficient \\
\hline $\bar{R}_{\mathrm{c}}$ & Dimensionless core circumradius & $E_{\mathrm{c}}, E_{\mathrm{w}}$ & Young modulus of core, wing \\
\hline $\bar{R}_{\mathrm{w}}$ & Dimensionless core circumradius & $\nu_{\mathrm{c}}, \nu_{\mathrm{w}}$ & Poisson coefficient of core, wing \\
\hline \multicolumn{2}{|c|}{ Geometric parameters } & \multicolumn{2}{|c|}{ Other parameters } \\
\hline $2 \pi p$ & Pitch of the helix & $\mathcal{E}_{S}$ & Total elastic energy \\
\hline$A_{\mathrm{c}}, A_{\mathrm{w}}$ & Cross-sectional area of core, wing & $\mathcal{E}_{\text {core }}$ & Elastic energy of the core \\
\hline$\epsilon_{\mathrm{c}}, \epsilon_{\mathrm{w}}$ & Longitudinal strain of core, wing & $\bar{\epsilon}_{\text {crit }}$ & Strain of core at instability \\
\hline$\epsilon_{\mathrm{c}}^{*}, \epsilon_{\mathrm{w}}^{*}$ & Imposed eigenstrain on core, wing & $\mathcal{E}_{\text {wing }}$ & Elastic energy of one wing \\
\hline$I_{\mathrm{c}}, I_{\mathrm{w}}$ & Second moment of area of core, wing & $S_{\text {crit }}$ & External stimulus at instability \\
\hline$J_{\mathrm{c}}, J_{\mathrm{w}}$ & Torsion constant of core, wing & $S$ & External stimulus \\
\hline
\end{tabular}

of area and torsion constant ${ }^{2}$ of its cross-section. The action of the stimulus is described as an "eigenstretching" of the core and the wings via the imposed eigenstrain $\epsilon^{*}$, controlled by the external stimulus $S$ via $\epsilon_{\bullet}^{*}=\alpha_{\bullet} S$ where $\alpha_{\bullet}$ is the susceptibility to the external stimulus $S(\bullet \in\{\mathrm{c}, \mathrm{w}\})$. For example, the external stimulus can be a variation of temperature $S=\Delta T$ and $\alpha$ would then be the coefficient of thermal expansion. According to our kinematical scenario, the energy of the core $\mathcal{E}_{\text {core }}$ is a function of its stretching $\epsilon_{\mathrm{c}}$ and its torsion $\tau_{\mathrm{c}}$, and the energy of each wing $\mathcal{E}_{\text {wing }}$ is a function of its stretching $\epsilon_{\mathrm{w}}$, its curvature bending $\kappa_{\mathrm{w}}$ and its torsion $\tau_{\mathrm{w}}$. Because of the kinematical assumptions, the quantities $\epsilon_{\mathrm{c}}, \tau_{\mathrm{c}}, \epsilon_{\mathrm{w}}, \kappa_{\mathrm{w}}$ and $\tau_{\mathrm{w}}$ are constant along the twister's main axis. Considering isotropic materials $\left(\mu_{\bullet}=E_{\bullet} / 2\left(1+\nu_{\bullet}\right)\right)$, we have:

$$
\mathcal{E}_{\text {core }}\left(\epsilon_{\mathrm{c}}, \tau_{\mathrm{c}}\right)=\frac{L_{\mathrm{c}} E_{\mathrm{c}}}{2}\left(A_{\mathrm{c}}\left(\epsilon_{\mathrm{c}}-\epsilon_{\mathrm{c}}^{*}\right)^{2}+\frac{J_{\mathrm{c}}}{2\left(1+\nu_{\mathrm{c}}\right)} \tau_{\mathrm{c}}^{2}\right),
$$

and

$$
\mathcal{E}_{\mathrm{wing}}\left(\epsilon_{\mathrm{w}}, \kappa_{\mathrm{w}}, \tau_{\mathrm{w}}\right)=\frac{L_{\mathrm{w}} E E_{\mathrm{w}}}{2}\left(A_{\mathrm{w}}\left(\epsilon_{\mathrm{w}}-\epsilon_{\mathrm{w}}^{*}\right)^{2}+I_{\mathrm{w}} \kappa_{\mathrm{w}}^{2}+\frac{J_{\mathrm{w}}}{2\left(1+\nu_{\mathrm{w}}\right)} \tau_{\mathrm{w}}^{2}\right),
$$

where $L_{\mathrm{c}}$ and $L_{\mathrm{w}}$ denote the length of the center line of the core and wing respectively. The total energy $\mathcal{E}_{S}=\mathcal{E}_{\text {core }}+n \mathcal{E}_{\text {wing }}$ together with the proposed kinematics embed enough "information" to achieve the targeted twisting upon activation above a stimulus threshold $S_{\text {crit }}$. To support this claim, $\mathcal{E}_{S}$ is now going to be derived in the case of the previously introduced polygonial geometries.

The chosen kinematics can first be used to reduce the number of parameters. Introducing the dimensionless torsion of the core $\bar{\tau}=r \tau_{\mathrm{c}}=r \mathrm{~d} \theta / \mathrm{d} z=r / p$ and given that the arclength variation of an helix of radius $r$ and pitch $p$ is related to the variation of its azimuthal angle along the transversal

2 The torsion constant should not be confused with the polar moment of inertia, which are only equal for circular shapes. More details are given in appendix A.4. 
direction $\theta$ by $\mathrm{d} s=\sqrt{r^{2}+p^{2}} \mathrm{~d} \theta$, the length of the core $\left(L_{\mathrm{c}}\right)$ and the length of a wing $\left(L_{\mathrm{w}}\right)$ are related by:

$$
L_{\mathrm{c}}=\int_{0}^{L_{\mathrm{c}}} \mathrm{d} z=p \int_{0}^{\frac{L_{\mathrm{c}}}{p}} \mathrm{~d} \theta=\frac{p}{\sqrt{r^{2}+p^{2}}} \int_{0}^{L_{\mathrm{w}}} \mathrm{d} s=\frac{L_{\mathrm{w}}}{\sqrt{1+\bar{\tau}^{2}}} .
$$

The relation between $L_{\mathrm{c}}$ and $L_{\mathrm{w}}$ therefore only involves $\bar{\tau}$. In line with the notation $\bar{\tau}$ for the dimensionless torsion of the core, we introduce the notation $\bar{\epsilon}=\epsilon_{\mathrm{c}}$ for the dimensionless stretching of the core. In the framework of large transformations [3, sec. 2.2], the stretching of the wings $\left(\epsilon_{\mathrm{w}}\right)$ and the stretching of the core $\left(\epsilon_{\mathrm{c}}\right)$ are defined as:

$$
\epsilon_{\mathrm{w}}=\frac{\left(\frac{L_{\mathrm{w}}}{L_{0}}\right)^{2}-1}{2} \text { and } \bar{\epsilon}=\epsilon_{\mathrm{c}}=\frac{\left(\frac{L_{\mathrm{c}}}{L_{0}}\right)^{2}-1}{2},
$$

where $L_{0}$ denotes the initial length of the twister. If $\left|L_{\bullet}-L_{0}\right| \ll L_{0}$, this reduces to $\epsilon_{\bullet}=$ $\left(L_{\bullet}-L_{0}\right) / L_{0}$. Using eq. (2.4) yields the following relation between $\epsilon_{\mathrm{c}}$ and $\epsilon_{\mathrm{w}}$ :

$$
\epsilon_{\mathrm{w}}=\frac{\left(\frac{L_{\mathrm{w}}}{L_{0}}\right)^{2}-1}{2}=\frac{\left(1+\bar{\tau}^{2}\right)\left(\frac{L_{\mathrm{c}}}{L_{0}}\right)^{2}-1}{2}=\bar{\epsilon}\left(1+\bar{\tau}^{2}\right)+\frac{1}{2} \bar{\tau}^{2} .
$$

Assuming perfect attachment between the wings and the core (as required by the compatibility of strain before delamination), the material curvature and torsion of the wings can be assimilated to the geometric curvature and torsion of their center line helix defined by $\kappa_{\mathrm{w}}=r /\left(r^{2}+p^{2}\right)$ and $\tau_{\mathrm{w}}=p /\left(r^{2}+p^{2}\right)$ respectively. In their chosen dimensionless form, the material curvature and torsion of the wings are:

$$
\bar{\kappa}_{\mathrm{w}}=r \kappa_{\mathrm{w}}=\frac{\bar{\tau}^{2}}{1+\bar{\tau}^{2}}, \quad \overline{\tau_{\mathrm{w}}}=r \tau_{\mathrm{w}}=\frac{\bar{\tau}}{1+\bar{\tau}^{2}} .
$$

Finally, the material curvature of the core is zero $\left(\kappa_{\mathrm{c}}=0\right.$, straight line). The proposed helical morphing scenario is hence such that all geometric variables (lengths, stretchings, curvatures and torsions) can be expressed as functions of $L_{\mathrm{c}}, \bar{\epsilon}$ and $\bar{\tau}$ using eq. (2.4), eq. (2.6), eq. (2.7). The configuration of a $n$-twister for a given stimulus, which is a priori parametrized by the eight geometric quantities $L_{\mathrm{c}}, L_{\mathrm{w}}, \epsilon_{\mathrm{c}}, \epsilon_{\mathrm{w}}, \kappa_{\mathrm{c}}, \kappa_{\mathrm{w}}, \tau_{\mathrm{c}}$ and $\tau_{\mathrm{w}}$, is thus entirely determined by the stretching and torsion of its core $(\bar{\epsilon}$ and $\bar{\tau})$ - and of course by the initial geometry governed by $n, L_{0}, R_{\mathrm{c}}$ and $R_{\mathrm{w}}$ as well as the material properties $E_{\mathrm{c}}, E_{\mathrm{w}}, \nu_{\mathrm{c}}, \nu_{\mathrm{w}}, \alpha_{\mathrm{c}}$ and $\alpha_{\mathrm{w}}$. This implies that the elastic energy of the whole twister is a function of $\bar{\epsilon}$ and $\bar{\tau}$ only. Using the terminology of catastrophe theory [10], $n, L_{0}, R_{\mathrm{c}}$ and $R_{\mathrm{w}}$ as well as $E_{\mathrm{c}}, E_{\mathrm{w}}, \nu_{\mathrm{c}}, \nu_{\mathrm{w}}, \alpha_{\mathrm{c}}$ and $\alpha_{\mathrm{w}}$ are control parameters, while $\bar{\epsilon}$ and $\bar{\tau}$ are state variables.

The energy of one wing becomes:

$$
\begin{aligned}
\mathcal{E}_{\text {wing }}(\bar{\epsilon}, \bar{\tau})=\sqrt{1+\bar{\tau}^{2}} \frac{L_{\mathrm{c}} E_{\mathrm{w}}}{2}( & A_{\mathrm{w}}\left(\bar{\epsilon}\left(1+\bar{\tau}^{2}\right)+\frac{1}{2} \bar{\tau}^{2}-\epsilon_{\mathrm{w}}^{*}\right)^{2} \\
& \left.+I_{\mathrm{w}}\left(\frac{\bar{\tau}^{2}}{r\left(1+\bar{\tau}^{2}\right)}\right)^{2}+\frac{J_{\mathrm{w}}}{2\left(1+\nu_{\mathrm{w}}\right)}\left(\frac{\bar{\tau}}{r\left(1+\bar{\tau}^{2}\right)}\right)^{2}\right) .
\end{aligned}
$$

Using, for sake of simplicity, the additional assumption that the two materials have the same Poisson 
ratio $\left(\nu_{\mathrm{c}}=\nu_{\mathrm{w}}=\nu\right)$, the energy of a $n$-twister consisting of one core and $n$ wings is such that:

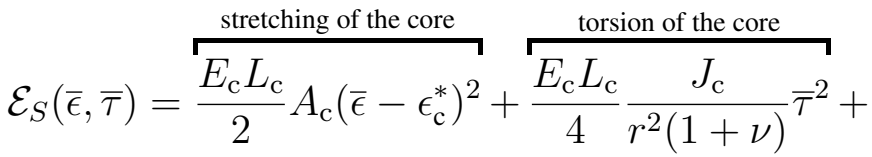

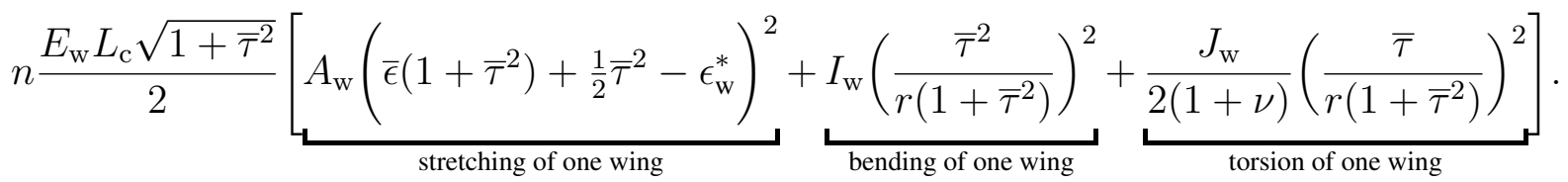

The area, second moment of area and torsion constant are given by $A_{\bullet}=A_{0} R_{\bullet}^{2}, I_{\bullet}=I_{0} R_{\bullet}^{4}$ and $J_{\bullet}=J_{0} R_{\bullet}^{4}$, where $A_{0}, I_{0}$ and $J_{0}$ are geometric parameters depending only on the shape (governed by $n$ ). The analytical expressions of $A_{0}(n)$ and $I_{0}(n)$ are derived in appendix $\mathrm{A}$, where the approximated values of $J_{0}$ for $n \in\{3,4,6\}$ are also given. It is worth noting that with the chosen kinematics, each wing deforms w.r.t. its neutral axis, not the twister's, so that each second moment of area needs to be calculated for an axis passing through the centroid of its polygon, and not the centroid of the twister. Additionally, second moment of inertia of sections with at least three axes of symmetry do not depend on the direction of the axis they are calculated with [8]. This holds in particular for all regular polygons and simplifies the calculation in the present case.

Observing that $L_{\mathrm{c}}=L_{0} \sqrt{1+2 \bar{\epsilon}}$ (from eq. (2.5)), adding $S$ as an explicit parameter and introducing the stiffness ratio $\bar{E}=E_{\mathrm{c}} / E_{\mathrm{w}}$ as well as the dimensionless core and wing circumradii $\bar{R}_{\mathrm{c}}=R_{\mathrm{c}} / r$ and $\bar{R}_{\mathrm{w}}=R_{\mathrm{w}} / r$, the dimensionless scaled elastic energy $\left(\overline{\mathcal{E}}_{S}=2 \mathcal{E}_{S} /\left(L_{0} E_{\mathrm{w}} R_{\mathrm{w}}^{2}\right)\right)$ is finally given by:

$$
\begin{aligned}
& \overline{\mathcal{E}}_{S}(\bar{\epsilon}, \bar{\tau})=\sqrt{1+2 \bar{\epsilon}}\left[\bar{E} \frac{\bar{R}_{\mathrm{c}}^{2}}{\bar{R}_{\mathrm{w}}^{2}}\left(A_{0}\left(\bar{\epsilon}-\alpha_{\mathrm{c}} S\right)^{2}+\frac{\bar{R}_{\mathrm{c}}^{2}}{2} \frac{J_{0}}{(1+\nu)} \bar{\tau}^{2}\right)+\right. \\
& \left.n \sqrt{1+\bar{\tau}^{2}}\left(A_{0}\left(\bar{\epsilon}\left(1+\bar{\tau}^{2}\right)+\frac{1}{2} \bar{\tau}^{2}-\alpha_{\mathrm{w}} S\right)^{2}+\bar{R}_{\mathrm{w}}^{2}\left(I_{0} \frac{\bar{\tau}^{4}}{\left(1+\bar{\tau}^{2}\right)^{2}}+\frac{J_{0}}{2(1+\nu)} \frac{\bar{\tau}^{2}}{\left(1+\bar{\tau}^{2}\right)^{2}}\right)\right)\right] .
\end{aligned}
$$

3. Characterisation of the twister's response The expression of the energy (eq. (2.10)) can be used to compute the response of a twister to a stimulus $S$ by minimising $\overline{\mathcal{E}}_{S}$ with respect to $\bar{\epsilon}$ and $\bar{\tau}$. This could be done numerically, but the optimisation problem can be simplified with the use of optimality conditions. Necessarily, an equilibrium point $(\bar{\epsilon}, \bar{\tau})$ is a stationary point of $\overline{\mathcal{E}}_{S}$, that is $\nabla \overline{\mathcal{E}}_{S}=0$ or equivalently:

$$
\left\{\begin{array}{l}
\partial_{\bar{\epsilon}} \overline{\mathcal{E}}_{S}(\bar{\epsilon}, \bar{\tau})=0 \\
\partial_{\bar{\tau}} \overline{\mathcal{E}}_{S}(\bar{\epsilon}, \bar{\tau})=0
\end{array}\right.
$$

The evolution of a twister starting from its resting position $(\bar{\epsilon}, \bar{\tau})=(0,0)$ for $S=0$ is now considered. Since eq. (3.2) is always satisfied for $\bar{\tau}=0$ (because the energy is an even function of $\bar{\tau}$ ), the response of a twister to the stimulus $S$ is governed ${ }^{3}$ only by eq. (3.1), as long as no critical point of $\partial_{\bar{\tau}} \overline{\mathcal{E}}_{S}$ is reached. Equation (3.1) with $\bar{\tau}=0$ constitutes the governing law of the stretching phase, by providing the strain $\bar{\epsilon}$ induced by the stimulus. In order to perform a qualitative analysis, $\partial_{\bar{\epsilon}} \overline{\mathcal{E}}_{S}(\bar{\epsilon}, 0)=0$ can be approximated by using a first-order Taylor expansion in $\bar{\epsilon}$ in the

\footnotetext{
${ }^{3}$ This necessary condition is also a sufficient condition because the energy is locally convex at $(\bar{\epsilon}, \bar{\tau})=(0,0)$ for $S=0$ (the determinant of the Hessian matrix of $\overline{\mathcal{E}}_{0}(0,0)$ is positive), and because it remains convex until $(\bar{\epsilon}, \bar{\tau})$ becomes a critical point of $\partial_{\bar{\tau}} \overline{\mathcal{E}}_{S}$.
} 
neighbourhood of 0 (legitimized by the small strains assumption $\bar{\epsilon} \ll 1$ ). Expressing $\bar{\epsilon}$ in terms of $S$ yields:

$$
\bar{\epsilon} \approx \frac{\alpha_{\mathrm{w}} n+\alpha_{\mathrm{c}} \overline{E R}^{2}}{n+\overline{E R}^{2}} S=\left(\alpha_{\mathrm{w}}-\frac{\Delta \alpha}{1+n /\left(\overline{E R}^{2}\right)}\right) S,
$$

where $\bar{R}=R_{\mathrm{c}} / R_{\mathrm{w}}=\bar{R}_{\mathrm{c}} / \bar{R}_{\mathrm{w}}$ is the size ratio and $\Delta \alpha=\alpha_{\mathrm{w}}-\alpha_{\mathrm{c}}$ is the differential expansion coefficient. The strains of core and wings are the same in the stretching phase $\left(\epsilon_{\mathrm{w}}=\epsilon_{\mathrm{c}}=\bar{\epsilon}\right.$ from eq. (2.6) with $\bar{\tau}=0$ ) and can be approximated by eq. (3.3). The core is not twisting $\left(\tau_{\mathrm{c}}=0\right)$ and the wings are thus neither bending nor twisting ( $\tau_{\mathrm{w}}=\kappa_{\mathrm{w}}=0$ from eq. (2.7) with $\bar{\tau}=0$ ). Equation (3.3) shows that when $E_{\mathrm{c}} \rightarrow \infty$ or $R_{\mathrm{c}} \rightarrow \infty$, the behaviour is dominated by the core $\left(\bar{\epsilon} \sim \alpha_{\mathrm{c}} S\right)$, which is relevant. On the contrary, when $n \rightarrow \infty$, the behavior follows the stretching of the wings $\left(\bar{\epsilon} \sim \alpha_{\mathrm{w}} S\right)$. We can also check that the stretching increases with $\alpha_{\mathrm{w}}$, as expected.

Now that the stretching phase has been characterized, the question is whether the proposed candidate is actually a twister. In other words, is there a value $S_{\text {crit }}$ such that the system starts twisting when the stimulus reaches $S_{\text {crit }}$ ? This boils down to whether $\partial_{\bar{\tau}} \overline{\mathcal{E}}_{S}(\bar{\epsilon}, 0)$ reaches a critical point, i.e.:

$$
\left\{\begin{array}{l}
\partial_{\bar{\tau} \bar{\epsilon}}^{2} \overline{\mathcal{E}}_{S}(\bar{\epsilon}, 0)=0 \\
\partial_{\bar{\tau} \bar{\tau}}^{2} \overline{\mathcal{E}}_{S}(\bar{\epsilon}, 0)=0
\end{array}\right.
$$

for some $\bar{\epsilon}$. Equation (3.4) is satisfied for all $\bar{\epsilon}$ and $S$, because it equals $\partial_{\bar{\epsilon}}^{2} \overline{\mathcal{E}}_{S}$ which is the derivative w.r.t. $\bar{\tau}$ of an even function of $\bar{\tau}$. The existence of a critical point is thus governed by eq. (3.1) (stretching phase) and eq. (3.5) (critical point). Though very simple to solve numerically, this reduces to a system of two independent quadratic equations in $\bar{\epsilon}$ and $S$, which does not have closed-form solutions that can be reasonably apprehended. For the sensitivity analysis to come in section 4, we linearize eq. (3.1) as previously, leading to eq. (3.3). This expression of $\bar{\epsilon}$ is then injected into eq. (3.5) which provides the following expression of the critical stimulus:

$$
S_{\text {crit }} \approx\left(n+\overline{E R}^{2}\right) \frac{\sqrt{1+A B\left(n+\overline{E R}^{4}\right) \bar{R}_{\mathrm{c}}^{2}}-1}{A},
$$

with $A=4 \alpha_{\mathrm{w}} n+\left(5 \alpha_{\mathrm{c}}-\alpha_{\mathrm{w}}\right) \overline{E R}^{2}=4 \alpha_{\mathrm{w}}\left(n+\overline{E R}^{2}\right)-5 \Delta \alpha \overline{E R}^{2}$ and $B=J_{0}\left(A_{0} \Delta \alpha n(1+\nu) \overline{E R}^{4}\right)^{-1}$. The corresponding $\bar{\epsilon}_{\text {crit }}$ can be easily retrieved using eq. (3.3):

$$
\bar{\epsilon}_{\mathrm{crit}} \approx\left(\alpha_{\mathrm{w}} n+\alpha_{\mathrm{c}} \overline{E R}^{2}\right) \frac{\sqrt{1+A B\left(n+\overline{E R}^{4}\right) \bar{R}_{\mathrm{c}}^{2}}-1}{A} .
$$

When $\alpha_{\mathrm{w}} \rightarrow \alpha_{\mathrm{c}}(\Delta \alpha \rightarrow 0), B \rightarrow \infty$ and we have $S_{\text {crit }} \rightarrow \infty$ as well as $\bar{\epsilon}_{\text {crit }} \rightarrow \infty$, meaning that the critical point is not reachable. This is relevant: there is no differential expansion, hence no twist. Cases $\bar{E} \rightarrow \infty$ or $\bar{E} \rightarrow 0$ (core or wings become infinitely rigid) and $n \rightarrow \infty$ (axisymmetric structure) also lead to $S_{\text {crit }} \rightarrow \infty$ and $\bar{\epsilon}_{\text {crit }} \rightarrow \infty$, as expected. We insist that the approximation provided by eq. (3.6) does not formally prove the existence of a bifurcation: it is not excluded that the approximation of $S_{\text {crit }}$ exists for a given set of geometric and material parameters, while the exact system of equations has no solutions for the same set of parameters, meaning there is no critical point. The tedious case by case discussion would not bring much: what matters is that for a very large majority of parameters, i) there is a critical point and ii) it is well approximated by eq. (3.6) and eq. (3.7). Both i) and ii) will be illustrated in the next section, demonstrating that the proposed mechanical system is an actual twister.

The post-instability evolution $\left(S>S_{\text {crit }}\right)$ remains to be characterised. As for the stretching phase, the governing equations are eqs. (3.1) and (3.2). However, now $\bar{\tau} \neq 0$ so the second equation 
is not always satisfied: both equations are necessary ${ }^{4}$. Equations (3.1) and (3.2) can easily be solved numerically; they can also be approximated locally using Tayor expansions, but it is not useful for the discussion. More interesting is the limit value of $\bar{\tau}$ as the stimulus increases. Solving eq. (3.2) for $S$ and injecting the result into eq. (3.1) for $\bar{\epsilon} \rightarrow \infty$ leads to the asymptotic values of $\bar{\tau}$ :

$$
\lim _{\bar{\epsilon} \rightarrow \infty} \bar{\tau}= \pm \sqrt{\frac{\Delta \alpha}{\alpha_{\mathrm{c}}}} .
$$

This limit shows that as the stimulus increases in the strecthing $\&$ twisting regime, the torsion asymptotically reaches a value which only depends on the expansion coefficients $\alpha_{\mathrm{c}}$ and $\alpha_{\mathrm{w}}$. This provides a very simple way of estimating the maximum twist.

In this section, the governing equations of the stretching and stretching-twisting phases, summarized in table 1, have been given. They are used and illustrated in the following section.

\begin{tabular}{cccc}
\hline Regime & $\begin{array}{c}\text { Kinematical } \\
\text { scenario }\end{array}$ & $\begin{array}{c}\text { Governing } \\
\text { equation(s) }\end{array}$ & $\begin{array}{c}\text { Approximated } \\
\text { Solution }\end{array}$ \\
Stretching & $\left\{\begin{array}{l}\epsilon_{\mathrm{c}}=\epsilon_{\mathrm{w}}=\bar{\epsilon} \\
\tau_{\mathrm{c}}=\tau_{\mathrm{w}}=0\end{array}\right.$ & $\partial_{\bar{\epsilon}} \overline{\mathcal{E}}_{S}(\bar{\epsilon}, 0)=0$
\end{tabular}

Table 1: Summary of the equations governing the response of the twister to the stimulus $S$.

4. Results and discussion The governing equations of the twister in both stretching and stretching-twisting regimes have been provided and approximated in the previous section. These expressions are now used to design twisters in accordance with some technical specifications. The design possibilities are illustrated for four different twisters:

- Twister 0 (T0) is a reference twister with arbitrary parameters;

- Twister 1 (T1) should stretch more than T0 and twist with a smaller amplitude;

- Twister 2 (T2) should stretch less than T0 and twist with a larger amplitude;

- Twister 3 (T3) should stretch more than T0 and twist with a larger amplitude.

The sensitivity of $S_{\text {crit }}$ and $\bar{\epsilon}_{\text {crit }}$ to the parameters values is analysed using eqs. (3.6) and (3.7). The sign of the variation of $S_{\text {crit }}$ for a small change of the parameters around their reference values (see table 3, line T0) is reported in table 2. For the chosen reference values the sign of the variation of $\bar{\epsilon}_{\text {crit }}$ is the same as the sign of variation of $S_{\text {crit }}$. The relevance of the result of the sensitivity analysis is detailed for each parameter in the third column.

\footnotetext{
${ }^{4}$ For the stretching phase, there were one equation for two unknowns $(\bar{\tau}=0)$ : solutions are, generically, curves. For the twisting phase, there are two equations for the three unknows $\bar{\epsilon}, \bar{\tau}$ and $S$, so the solutions are also curves: the additional variable $\bar{\tau}$ is "compensated" by the additional equation $\partial_{\bar{\tau}} \overline{\mathcal{E}}_{S}$.
} 


\begin{tabular}{|c|c|c|}
\hline $\begin{array}{l}\text { Increasing } \\
\text { Parameter }\end{array}$ & $\begin{array}{l}\text { Variation of } \\
S_{\text {crit }} \text { and } \bar{\epsilon}_{\text {crit }}\end{array}$ & Comments on relevance \\
\hline$n$ & $\nearrow$ & $\begin{array}{l}\text { As } n \text { increases, the twister gets closer to an axisymmetric } \\
\text { structure, which does not twist. }\end{array}$ \\
\hline $\bar{E}$ & $\nearrow$ & $\begin{array}{l}\text { Increasing the stiffness of the core results in a higher resis- } \\
\text { tance to twist. }\end{array}$ \\
\hline$\nu$ & $\searrow$ & $\begin{array}{l}\text { When the Poisson's ratio increases, the twisting stiffness } \\
\text { decreases relatively to the stretching stiffness, thus lowering } \\
\text { the resistance to twist. }\end{array}$ \\
\hline $\bar{R}$ & $\nearrow$ & $\begin{array}{l}\text { Increasing the relative volume of the core results in a higher } \\
\text { resistance to twist. }\end{array}$ \\
\hline$\Delta \alpha=\alpha_{\mathrm{w}}-\alpha_{\mathrm{c}}$ & $\searrow$ & $\begin{array}{l}\text { Increasing the differential expansion pushes twisting to acti- } \\
\text { vate sooner. }\end{array}$ \\
\hline
\end{tabular}

Table 2: Variations of $S_{\text {crit }}$ and $\bar{\epsilon}_{\text {crit }}$ for small increases of the parameters around their reference values. An arrow $\nearrow$ for a parameter indicates that $S_{\text {crit }}$ and $\bar{\epsilon}_{\text {crit }}$ increases when the parameter increases, meaning that the twister becomes less favorable to twisting.

Table 2 serves as a guideline for the design of T1, T2 and T3 by adjusting the parameters of T0. T1 should i) twist for large value of $\bar{\epsilon}_{\text {crit }}$ and ii) the post-instability twisting amplitude should be small. Equation (3.8) shows ii) can only be achieved with a small difference of expansion coefficients $\Delta \alpha$, compared to $\alpha_{\mathrm{c}}$. Specification i) can be enhanced by increasing $\bar{E}$, for example. For T2, a similar reasoning leads to a reduction of $\bar{R}$ and an increase of $\Delta \alpha$ while T3 is set by increasing $\bar{E}$ as well as the differential expansion $\Delta \alpha$. The selected values are reported in table 3.

\begin{tabular}{cllllllll}
\hline Twister & $n$ & $\bar{E}$ & $\nu$ & $R_{\mathrm{c}}$ & $R_{\mathrm{w}}$ & $\alpha_{\mathrm{c}}$ & $\alpha_{\mathrm{w}}$ \\
$\mathrm{T} 0$ & 3 & 3 & 0 & 3 & 2 & 0.4 & 0.5 \\
$\mathrm{~T} 1$ & 3 & $\mathbf{4}$ & 0 & 3 & 2 & $\mathbf{0 . 4}$ & $\mathbf{0 . 4 1}$ \\
$\mathrm{T} 2$ & 3 & 3 & 0 & $\mathbf{1}$ & $\mathbf{1}$ & $\mathbf{0 . 5}$ & $\mathbf{1}$ \\
$\mathrm{T} 3$ & 3 & $\mathbf{3 0 0}$ & 0 & 3 & 2 & 0.4 & $\mathbf{0 . 8}$ \\
\hline
\end{tabular}

Table 3: Parameter values of the four twisters.

The critical values of the instability have been computed by solving equations (3.1) and (3.5) (see table 1) and are compared to the approximate equations provided in section 2. For all cases, such critical values exist: this means that the proposed model actually describes a twisting instability. Moreover, the approximations of $S_{\text {crit }}$ and $\bar{\epsilon}_{\text {crit }}$ are very close to the computed values, which validates eq. (3.6) and eq. (3.7), as well as the approximate description of the stretching phase.

The state evolution of a twister in response to an increasing stimulus results from the simple numerical resolution of the governing equations (cf. table 1). All numerical results were computed in Mathematica. Figure 3 depicts the results for each chosen twister. The asymptotes directly follow from eq. (3.8). For each case, the observed behaviour is consistent with the FEM simulations: stretching regime, then instability giving birth to a stretching-twisting regime. Branches are symmetric with respect to $\bar{\tau}$ but negative branches have not been represented. It is worth mentionning that, contrary to the small strain assumption, the strains around the twisting instability can be 


\begin{tabular}{|c|c|c|c|c|c|c|}
\hline \multirow{2}{*}{ Twister } & \multicolumn{2}{|c|}{ "Exact" value } & \multicolumn{2}{|c|}{ Approximation } & \multicolumn{2}{|c|}{ Relative error } \\
\hline & $S_{\text {crit }}$ & $\bar{\epsilon}_{\text {crit }}$ & $S_{\text {crit }}$ & $\bar{\epsilon}_{\text {crit }}$ & $S_{\text {crit }}$ & $\overline{\bar{\epsilon}_{\text {crit }}}$ \\
\hline T0 & 1.61 & 0.694 & 1.60 & 0.689 & $0.6 \%$ & $0.7 \%$ \\
\hline $\mathrm{T} 1$ & 6.83 & 2.75 & 6.84 & 2.75 & $0.1 \%$ & $0.0 \%$ \\
\hline $\mathrm{T} 2$ & 0.383 & 0.285 & 0.391 & 0.293 & $1.9 \%$ & $2.9 \%$ \\
\hline $\mathrm{T} 3$ & 8.72 & 3.50 & 8.72 & 3.50 & $0.0 \%$ & $0.0 \%$ \\
\hline
\end{tabular}

Table 4: Comparison of the critical values of $\bar{\epsilon}$ and $S$ causing bifurcations for the four twisters.

relatively high, thus contradicting Hook's law. However, the same qualitative behavior is expected with an adapted large deformation behavior law. In the end, the proposed mechanical model, though simple, proves to exhibit the targeted twisting behaviour.

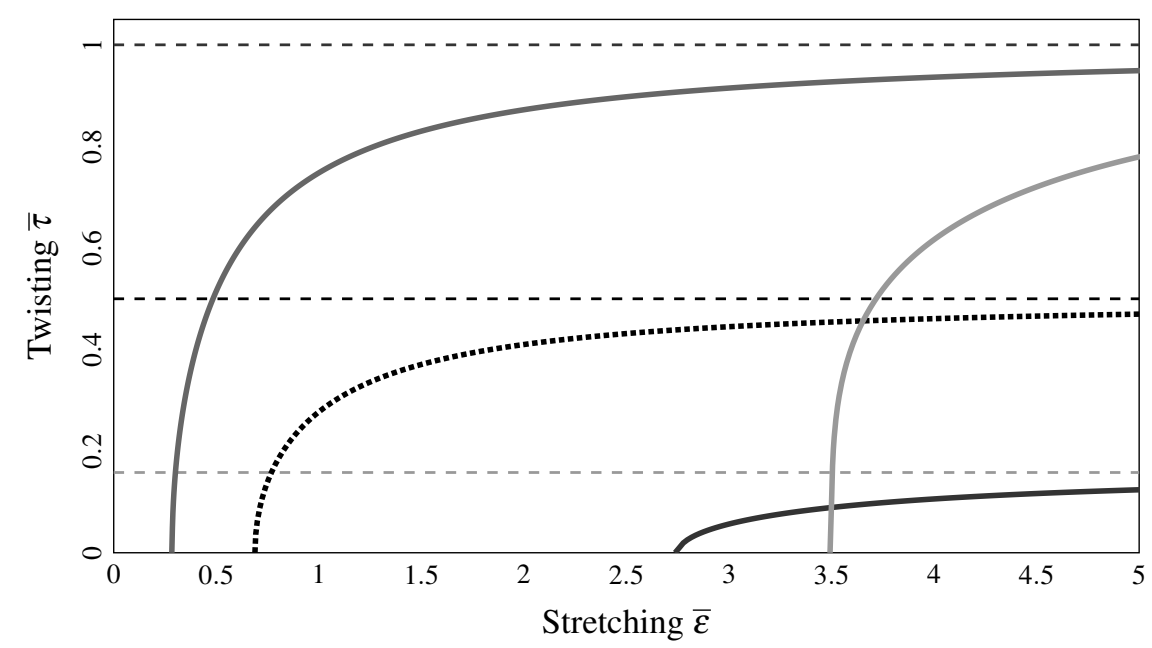

Figure 3: State evolutions of the twisters of table 3. […] T0, $[-] \mathrm{T} 1,[-] \mathrm{T} 2,[-] \mathrm{T} 3$. Dashed lines: asymptotes of $\bar{\tau}$ for $\bar{\epsilon} \rightarrow \infty$. Negative $\bar{\tau}$ have been omitted.

The critical value $S_{\text {crit }}$ plays the same role as the critical force in buckling theory: exactly as a beam subjected to an increasing normal force buckles in one direction when the force is sufficiently high, twisters subjected to an increasing stimulus twists in on direction $( \pm \bar{\tau})$ when the stimulus is sufficiently high. Animations of the four twisters are provided as supplementary material. Illustrations of the centroids of the core and one wing are provided in fig. 4 for the three twisters T1, $\mathrm{T} 2$ and $\mathrm{T} 3$.

Conclusion Inspired by Nature and based on symmetry considerations, a particular class of structures called twisters was proposed featuring open rotational symmetric cross-sections made out of regular polygons, where active wings are arranged around a passive core. A twister reacts to an external stimulus, such as a uniform temperature field, by differential expansion leading to a combination of stretching and twisting. A kinematical scenario based on FEM simulations was derived and used to construct a mechanical model with two state variables (the stretching strain and the twist). Governing equations were provided and it was shown that the evolution of the response to the stimulus featured a twisting instability: above a critical value of the stimulus $S_{\text {crit }}$, the twister's morphing behavior changes from a pure stretching regime to a stretching \& twisting regime. An approximation of $S_{\text {crit }}$ was derived and set the base for a simple sensitivity analysis. The latter was 


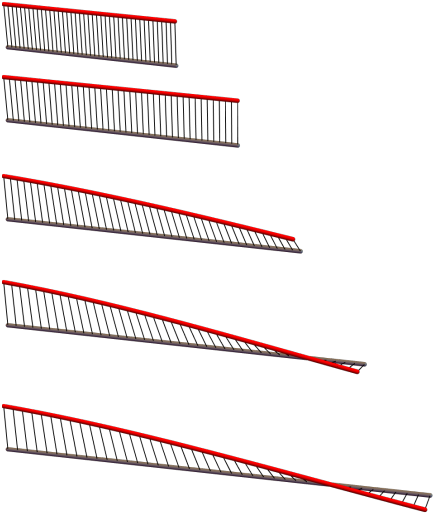

(a) T1: long stretching phase, small twisting amplitude.

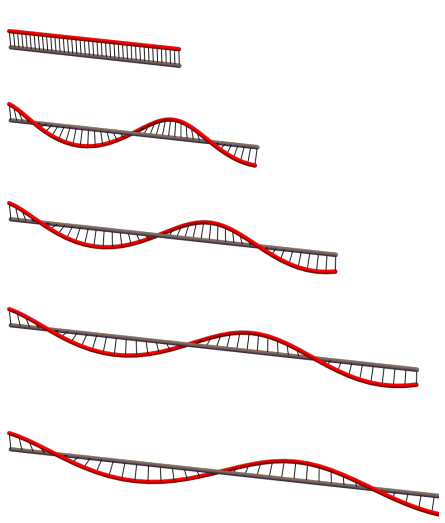

(b) T2: small stretching phase, large twisting amplitude.

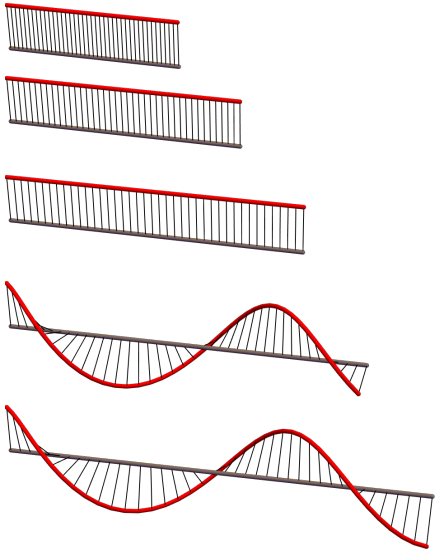

(c) T3: long stretching phase, large twisting amplitude.

Figure 4: Centroids of the core (light gray) and of one wing (red) for different levels of stimulus $S$. Each row corresponds to the same stimulus and all images share the same scale. Corresponding animations are available online.

used to choose the value of twisters parameters in accordance with some arbitrary specifications. Numerical simulations validated the design principle of the twister and the approximated value of $S_{\text {crit }}$ was shown to be very accurate. In the end, we now have a new simple structure, capable of responding to a uniform stimulus by stretching and twisting. The assumptions of constant twist and linear elastic materials were useful to derive understandable formulas and check their relevance. However, the presented methodology can be easily extended to more general kinematic scenarii or other constitutive laws.

By combining benders and twisters, it is theoretically possible to build rod-like morphers morphing into any curve in space as a stimulus increases. This new marterial architecture thus enriches the existing design space of passive actuators. The main remaining challenge now lies in the choice of appropriate material.

Author contributions statement S.T. conceived the study, formulated the mechanical model and contributed to about half of the manuscript. A.T. solved the mechanical model and contributed to about half of the manuscript. J.D, Y.B. and P.F. helped to concieve the study.

\section{References}

[1] Abraham, Yael, Carmen Tamburu, Eugenia Klein, John WC Dunlop, Peter Fratzl, Uri Raviv, Rivka ELBAUM. Tilted cellulose arrangement as a novel mechanism for hygroscopic coiling in the stork's bill awn. Journal of The Royal Society Interface 9(69):640-647, 2012.

[2] Armon, Shahaf, Efi Efrati, Raz Kupferman, Eran Sharon. Geometry and mechanics in the opening of chiral seed pods. Science 333(6050):1726-1730, 2011.

[3] Audoly, Basile, Yves Pomeau. Elasticity and geometry: from hair curls to the non-linear response of shells. Oxford University Press, 2010.

[4] CuRIE, P. Sur la symétrie dans les phénomènes physiques, symétrie d'un champ électrique et d'un champ magnétique. J. Phys. Theor. Appl. 3(1):393-415, 1894.

[5] DAwson, Colin, Julian FV VinCEnT, Anne-Marie RocCA. How pine cones open. Nature 390(6661):668-668, 1997.

[6] FRATZL, Peter, Friedrich G BARTH. Biomaterial systems for mechanosensing and actuation. Nature 462(7272):442448, 2009.

[7] MurA, T. Micromechanics of defects in solids,(1982). Martinus Nijhoff, 1982.

[8] Soerjadi, R. On the Computation of the Moments of a Polygon, with Some Applications. Stevin Laboratory, 1968. 
[9] STRUCTX. Structural shapes. 2017.

URL: http://www.structx.com/geometric\%5C_properties.html.

[10] Thom, René, David Herbert FowLER. Structural Stability and Morphogenesis: An Outline of a General Theory of Models 1St English Ed. Benjami, 1975.

[11] Timoshenko, S et al. Analysis of bi-metal thermostats. J. Opt. Soc. Am 11(3):233-255, 1925.

[12] TurCaud, Sébastien, Lorenzo Guiducci, Peter Fratzl, Yves JM BRÉchet, John WC Dunlop. An excursion into the design space of biomimetic architectured biphasic actuators. International Journal of Materials Research 102(6):607-612, 2011.

\section{A. Geometric relationships}

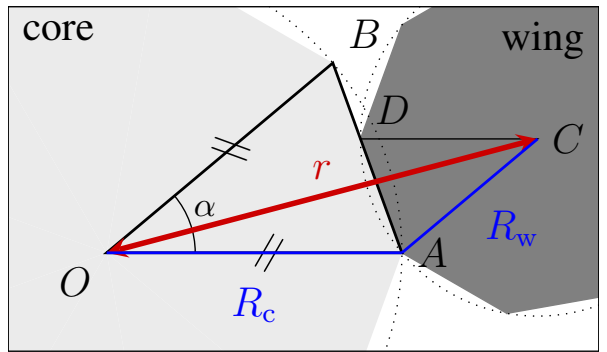

Figure 5: Cross-section of a $n$-twister and related notations.

A.1. Relation between $r, R_{\mathrm{c}}$ and $R_{\mathrm{W}}$ Geometric relationships can be easily derived by considering triangular slices of $n$-sided regular polygons, see fig. 5. Using elementary geometry:

$r^{2}=\|\overrightarrow{O C}\|=\|\overrightarrow{O A}+\overrightarrow{A C}\|=\left\|R_{\mathrm{c}}(1,0)+R_{\mathrm{w}}(\cos (\alpha), \sin (\alpha))\right\|=\left(R_{\mathrm{c}}+R_{\mathrm{w}} \cos (\alpha)\right)^{2}+\left(R_{\mathrm{w}} \sin (\alpha)\right)^{2}$, so, with $\bar{R}=R_{\mathrm{c}} / R_{\mathrm{w}}$ and since $\alpha=2 \pi / n$, we have:

$$
r=\sqrt{R_{\mathrm{c}}^{2}+2 R_{\mathrm{c}} R_{\mathrm{w}} \cos \left(\frac{2 \pi}{n}\right)+R_{\mathrm{w}}^{2}}=R_{\mathrm{w}} \sqrt{1+2 \bar{R} \cos \left(\frac{2 \pi}{n}\right)+\bar{R}^{2}} .
$$

A.2. Expression of $A_{0}(n)$ The areas of the core and of one wing are given by $A_{\mathrm{c}}=A_{0}(n) R_{\mathrm{c}}^{2}$ and $A_{\mathrm{w}}=A_{0}(n) R_{\mathrm{w}}^{2}$ where $A_{0}(n)$ is the area of a $n$-sided regular polygon of unit circumradius. Considering the triangle above, it comes directly:

$$
A_{\mathrm{c}}(n)=\frac{1}{2} n \times O A \times O B \sin (\alpha)=\frac{n}{2} R_{\mathrm{c}}^{2} \sin (\alpha) .
$$

so finally $A_{0}(n)=\frac{1}{2} n \sin (2 \pi / n)$ (note that $A_{0} \rightarrow \pi$ when $n \rightarrow \infty$ : it's the area of a unit disk).

A.3. Expression of $I_{0}(n)$ Without loss of generality, the $n$-sided regular polygon is assumed to be such that the vertices $A_{k}$ have coordinates $(\cos (2 \pi k / n), \sin (2 \pi k / n))$, for $k \in\{1, \ldots, n\}$ and $A_{n+1}=A_{1}$. Then, the expression of the second moment of area with respect ${ }^{5}$ to the $y$-axis is given by [8]:

$$
I_{y}=\frac{1}{12} \sum_{k=1}^{n}\left(x_{k}^{2}+x_{k} x_{k+1}+x_{k+1}^{2}\right)\left(x_{k} y_{k+1}-x_{k+1} y_{k}\right) .
$$

Using trigonometric identities, this expression simplifies nicely. The right parenthesis becomes:

$$
x_{k} y_{k+1}-x_{k+1} y_{k}=\cos \left(\frac{2 k \pi}{n}\right) \sin \left(\frac{2(k+1) \pi}{n}\right)-\cos \left(\frac{2(k+1) \pi}{n}\right) \sin \left(\frac{2 k \pi}{n}\right)=\sin \left(\frac{2 \pi}{n}\right),
$$

\footnotetext{
${ }^{5}$ The second moment of area does not depend on the direction of the axis for regular polygonial sections.
} 
so it does not depend on $k$ and can factorize the sum. Then,

$$
\sum_{k=1}^{n} x_{k}^{2}=\sum_{k}^{n} x_{k+1}^{2}=\frac{1}{2} \sum_{k=1}^{n}\left(1+\cos \left(\frac{4 k \pi}{n}\right)\right)=\frac{n}{2},
$$

and using the identity $\cos (a) \cos (a+b)=(\cos (b)+\cos (2 a+b)) / 2$ it comes:

$$
\sum_{k=1}^{n} x_{k} x_{k+1}=\sum_{k=1}^{n} \frac{1}{2}\left(\cos \left(\frac{2 \pi}{n}\right)+\cos \left(\frac{4 k \pi}{n}+\frac{2 \pi}{n}\right)\right)=\frac{1}{2} n \cos \left(\frac{2 \pi}{n}\right) .
$$

Finally,

$$
I_{0}(n)=\frac{n}{12} \sin \left(\frac{2 \pi}{n}\right)\left(1+\frac{1}{2} \cos \left(\frac{2 \pi}{n}\right)\right) .
$$

$I_{0}(n) \rightarrow \frac{1}{4} \pi$ when $n \rightarrow \infty$, which corresponds to the second moment of area of a unit disk.

A.4. Expression of $J_{0}(n)$ In general, there is no closed-form expressions of the torsion constant $J_{0}(n)$. Following Saint-Venant's solution for uniform twist [3, sec. 3.4], one has to solve a Poisson equation on the potential $\chi(x, y)$ from which the shear strain vector field $-\epsilon_{y z} \mathbf{e}_{x}+\epsilon_{x z} \mathbf{e}_{y}$ with zero curl derives. By defining a rescaled potential $\bar{\chi}=\frac{\chi}{\tau}$ and having solved $\bar{\chi}$ for a particular cross-section, the torsion constant is given by:

$$
J=4 \iint_{D}\left(\bar{\chi}_{, x}^{2}+\bar{\chi}_{, y}^{2}\right) \mathrm{d} x \mathrm{~d} y
$$

\begin{tabular}{|c|c|c|c|c|}
\hline $\begin{array}{c}\text { side number } \\
n_{w}\end{array}$ & shape & $\begin{array}{c}\text { area } \\
A_{\bullet}=A_{0} R_{\bullet}^{2}\end{array}$ & $\begin{array}{c}\text { second moment of area } \\
\qquad I_{\bullet}=I_{0} R_{\bullet}^{4}\end{array}$ & $\begin{array}{l}\text { torsion constant } \\
\qquad J_{\bullet}=J_{0} R_{\bullet}^{4}\end{array}$ \\
\hline 3 & & $A_{0}=\frac{3 \sqrt{3}}{4} \approx 1.30$ & $I_{0}=\frac{3 \sqrt{3}}{32} \approx 0.162$ & $J_{0}=\frac{9 \sqrt{3}}{80} \approx 0.195$ \\
\hline 4 & & $A_{0}=2.00$ & $I_{0}=\frac{1}{3} \approx 0.333$ & $J_{0} \approx 0.563$ \\
\hline 6 & & $A_{0}=\frac{3 \sqrt{3}}{2} \approx 2.60$ & $I_{0}=\frac{5 \sqrt{3}}{16} \approx 0.541$ & $J_{0} \approx 1.04$ \\
\hline
\end{tabular}

A.5. Values for $n \in\{3,4,6\}$ Numerical approximations of $J_{0}(n)$ are given in table 5 for the considered geometries [9].

Table 5: Values of the geometric parameters of regular $n$-sided polygons $(n=3,4,6$ and $\bullet \in\{\mathrm{c}, \mathrm{w}\})$. 